\title{
Dual-tasking alleviated sleep deprivation disruption in visuomotor tracking: An fMRI study
}

\author{
Yunglin Gazes $^{\mathrm{a}}$, Brian C. Rakitin ${ }^{\mathrm{a}}$, Jason Steffener ${ }^{\mathrm{a}}$, Christian Habeck ${ }^{\mathrm{a}}$, Brady Butterfield ${ }^{\mathrm{a}}$, \\ Robert C. Basner ${ }^{\mathrm{b}}$, Claude Ghez $^{\mathrm{c}}$, and Yaakov Stern ${ }^{\mathrm{a}}$ \\ ${ }^{a}$ Cognitive Neuroscience Division, Taub Institute, Columbia University, 630 West 168th Street, P \\ \& S Box 16, New York, NY 10032, USA \\ bMedicine - Pulmonary, Allergy \& Critical Care, Columbia University, 630 West $168^{\text {th }}$ Street, $\mathrm{PH} 8$ \\ Center, Room 859, New York, New York 10032
}

'Department of Neurology and Neuroscience, Columbia University, 821 Psychiatric Institute Annex, 1051 Riverside Drive, New York, NY 10032, USA

\begin{abstract}
Effects of dual-responding on tracking performance after 49-hr of sleep deprivation (SD) were evaluated behaviorally and with functional magnetic resonance imaging (fMRI). Continuous visuomotor tracking was performed simultaneously with an intermittent color-matching visual detection task in which a pair of color-matched stimuli constituted a target and non-matches were non-targets. Tracking error means were binned time-locked to stimulus onset of the detection task in order to observe changes associated with dual-responding by comparing the error during targets and non-targets. Similar comparison was made with fMRI data. Our result showed that despite a significant increase in the overall tracking error post SD, from 20 pixels pre SD to 45 pixels post $\mathrm{SD}$, error decreased to a minimum of about 25 pixels 0 to $6 \mathrm{~s}$ after dual-response. Despite an overall reduced activation post SD, greater activation difference between targets and non-targets was found post SD in task-related regions, such as the left cerebellum, the left somatosensory cortex, the left extrastriate cortex, bilateral precuneus, the left middle frontal gyrus, and the left motor cortex. Our results suggest that dual-response helps to alleviate performance impairment usually associated with SD. The duration of the alleviation effect was on the order of seconds after dual-responding.
\end{abstract}

\section{Keywords}

Visuomotor tracking; Visual detection; Continuous wakefulness; Dual responses; Dual tasks; Prefrontal cortex

\footnotetext{
(C) 2012 Elsevier Inc. All rights reserved.

corresponding author: Yaakov Stern, Taub Institute, Columbia University, 630 West $168^{\text {th }}$ Street, P \& S Box 16, New York, NY 10032, ys11@ columbia.edu, Phone: 212-342-1350, Fax: 212-342-1838.

Publisher's Disclaimer: This is a PDF file of an unedited manuscript that has been accepted for publication. As a service to our customers we are providing this early version of the manuscript. The manuscript will undergo copyediting, typesetting, and review of the resulting proof before it is published in its final citable form. Please note that during the production process errors may be discovered which could affect the content, and all legal disclaimers that apply to the journal pertain.
} 


\section{Introduction}

As our society becomes more sleep deprived and more dependent on technology (National Science Foundation 2011 Sleep in America Poll), people are frequently multitasking while suffering from sleep deprivation. While sleep deprivation tends to lower our performance on tasks (Bell-McGinty et al., 2004; Donnell, 1969; Durmer \& Dinges, 2005; Habeck et al., 2004; Home, Anderson, \& Wilkinson, 1983; Pashler, 1994; Pilcher \& Huffcutt, 1996), a number of multi-task studies showed that performance was maintained after sleep deprivation (Pace-Schott et al., 2009; Pilcher, Band, Odle-Dusseau, \& Muth, 2007; Strangman, Thompson, Strauss, Marshburn, \& Sutton, 2005). In a comprehensive 28-hour sleep deprivation study, Pilcher et al. (2007) examined performance changes in tasks with a wide range of demand, from simple vigilance task such as the Psychomotor Vigilance Test (PVT) to the cognitively challenging Wombat, in which tracking task alternated with several bonus tasks. Performance on vigilance tests suffered after sleep deprivation but Wombat performance actually increased over the course of sleep deprivation sessions due to the learning effect. Strangman et al. (2005) reported that, in addition to maintained performance on a dual-joystick controlled navigation task, regions with increased as well as regions with decreased brain activation were found after sleep deprivation even though, with the exception of studies such as Drummond et al. (2000), traditional sleep deprivation studies generally showed only decreased brain activation with performance deficits (Chuah, Venkatraman, Dinges, \& Chee, 2006; Drummond et al., 1999; Thomas et al., 2000).

The mechanism underlying multi-tasking has been studied extensively using dual-tasking paradigms (Brown, 1997; Jolicoeur, 1999; Pashler, 1994)in which performance interference has been reported in one or both tasks due to the limited resources that are shared by the tasks (Brown, 1997; Jolicoeur, 1999; Pashler, 1994). If both dual-tasking and sleep deprivation have negative effects on performance, combining the two factors should have even more detrimental effects. Thus it seems contradictory that under sleep deprivation stress, dual-tasking actually protected performance from SD effects in comparison to performance on single-tasks. However, not all multi-task tracking showed maintained performance after SD. Caldwell et al. (1998) found a time-on-task post SD effect: performance worsened as the time spent on the task increased. The task used by the study was called the multi-attribute task battery (MATB), which consisted of a primary tracking task and three other detection tasks, one audio and two visual.

In a previous study, we examined a task similar to MATB but with only one detection task instead of three (Gazes et al., 2010). Behaviorally and with fMRI, we examined the effect of sleep deprivation on the MATB-like task to gain a better understanding of why a MATBlike task shows SD impairment but tasks used by Pilcher et al. and Strongman et al. do not. Tracking error means were binned so that they were time-locked to the stimulus onsets of the detection task to observe temporal changes in tracking relative to the secondary task. Understanding the discrepancy among multi-task sleep deprivation studies by examining the interaction between SD and dual-tasking may pinpoint important cognitive mechanisms that can help to resist the debilitating sleep deprivation effects. Specifically, based on our finding from Gazes et al. (2010), the most interference occurs in dual-responding for our task, if dual-tasking interacts with SD to form a protective effect on performance under SD, then the protective effect should be most pronounced during dual-responses.

\section{Experimental Procedure}

\subsection{Participants}

Twenty-four healthy participants completed the sleep deprivation protocol. Nineteen participants (11 males, 8 females), between the ages of 20 and 34 years (age $=23 \pm 3.6$ 
years), were included in the analyses. Four participants were excluded due to equipment problems and one participant was excluded based on a pre SD root-mean-square tracking error of greater than three standard deviations above the mean. All participants were righthanded and carefully screened to ensure that they had no history of medical, psychiatric, neurological or sleep disorder. Habitual coffee drinkers (1 cup or more coffee per day) were excluded from the study. Participants maintained a sleep log for 2 weeks prior to study; the average amount of sleep per participant per day was $8.5 \mathrm{~h}$. Mean within-subjects variability across the 14 days prior to the experiment gave a mean standard deviation of $1.3 \mathrm{~h}$. Sleep data was missing for two participants. Participants were instructed to stop drinking caffeine $24 \mathrm{~h}$ prior to study participation and for the duration of the study. All participants passed substance abuse screening tests. Participants were supervised at all times, and polysomnographic monitoring confirmed that they remained awake during the sleep deprivation period. Informed consent, as approved by the Internal Review Board of the College of Physicians and Surgeons of Columbia University, was obtained prior to study participation and after the nature and risks of the study were explained. Participants were paid for their participation in the study.

\subsection{Study protocol}

Participants were admitted into the hospital on a Monday and received a full night of rest (> $8 \mathrm{~h}$ ). Research personnel arrived at participant's room at 9 AM. The initial test scan occurred at $10 \mathrm{AM}$ (pre SD) Tuesday morning, and the follow-up scan occurred at the same time $48 \mathrm{~h}$ later (post SD) to eliminate confounding circadian effects, yielding approximately $49 \mathrm{~h}$ of prolonged wakefulness. The tracking study was performed as part of a larger experiment in which repetitive transcranial magnetic stimulation (rTMS) was administered twice on both Tuesday and Wednesday. Regions that received rTMS were based on a set of cortical regions identified by Habeck et al. (2004) to be associated with performance in a second task studied in the experiment, and thus the manipulation was not targeted at the tracking task. None of the effects of interest both behaviorally and in neuroimaging discussed in this paper showed an interaction with TMS manipulation (SD $\times$ TMS: $F(1,17)=0.200, p=$ $0.660 ; \mathrm{SD} \times$ Single/Dual Condition $\times$ TMS: $F(1,17)=1.99, p=0.177 ; \mathrm{SD} \times$ Single/Dual Condition $\times$ Target/Nontarget $\times$ TMS: $F(1,17)=1.41, p=0.252)$.

\subsection{Task}

All participants were tested in both the single- and the dual-task conditions. The single-task condition was added as a control condition in which participants performed only a visuomotor compensatory tracking task. In the dual-task condition, participants also performed a color matching visual detection task. For the continuous visuomotor tracking, a white plus sign constituted the reference position and was drawn fixed at the center of the screen (see Figure 1). At trial onset the cursor was aligned with the crosshair. At each update, a continuously varying pseudorandom perturbation was added to the previous $\mathrm{x}$ and $y$ coordinates of the cursor. This had the effect of moving the cursor away from the center as though an unpredictable force had been applied to the participant's hand. The task was then to maintain the cursor position aligned with the reference position (the plus sign) and to counteract the perturbation force using the right hand to control a joystick. The cursor position at each update was further determined by the net sum of all previous joystick movements such that a 2 unit movement in the positive $\times$ direction would continue to push the cursor to the right by 2 units until a joystick movement in the negative $\times$ direction of equal magnitude was made. Movements in the $\mathrm{x}$ - and $\mathrm{y}$-axis were calculated independently. The cursor was restricted to within a radius of 100 pixels from the reference position, thus setting a maximum distance of 100 pixels in any direction. If the cursor reached the maximum distance of 100 pixels, it remained at the same location until the participant moved it away. 
For both training and testing, each tracking trial lasted $126 \mathrm{~s}$. Training for the task lasted 27.4 minutes on 6 trials each of single- and dual-task conditions. Testing in the MRI scanner consisted of 3 blocks in which 2 trials of each condition were presented (4 trials per block) and randomly ordered across participants. Each testing block was 11.87 min with a $40 \mathrm{~s}$ rest interval and $2 \mathrm{~s}$ instruction screen at the start of each tracking trial. An additional $40 \mathrm{~s}$ rest interval was presented at the end of each block.

For the color-matching visual detection task, stimuli consisted of four circles arranged one in each of the four corners of the screen, appearing concurrently with the tracking cursor. The circles, which remained on the screen throughout the trial, start with one of the four possible colors (red, blue, green, and yellow), and may take on any of the four possible colors every few seconds, the frequency of which depends on the ITI as described below. Color match between diagonally positioned circles constituted a target to which participants were instructed to respond with a button press using their left index finger; any other circle color change was a non-target. Six targets were presented in every 2 min tracking trial. Mean inter-trial-interval (ITI) for target was $19.3 \pm 10.0 \mathrm{~s}$, and each target remained on the screen for $8 \mathrm{~s}$ regardless of response. Mean ITI for non-target was $3.86 \pm 2.44 \mathrm{~s}$. To maintain equal visual stimulation between the two conditions, detection stimuli were presented in the single-task condition, but participants were instructed to ignore the detection stimulus. Targets and non-targets were presented at the same frequency as in the dual task condition. For more details about the task, see Gazes et al. (2010).

The rest screen presented the same visual stimuli as in the test trial (instead of a blank rest screen) in order to subtract visual activation from all functional activations. The instruction screen consisted of the same visual presentation as the rest screen except for the condition cues. Instead of the word 'Rest' on the left and right side of the screen, the word 'Track' and 'Circles' were presented to cue single- and dual-task conditions, respectively. Condition cues remained on the screen throughout the tracking trials.

\section{4 fMRI data acquisition}

During each block of testing, 285 T2*-weighted images (BOLD images) (Ogawa et al., 1993), were acquired with an Intera 1.5-T Phillips MR scanner equipped with a 6-channel SENSE head coil (Pruessmann, Weiger, Scheidegger, \& Boesiger, 1999), using a gradient echo echo-planar (GE-EPI) sequence (time echo/time repetition $[\mathrm{TE} / \mathrm{TR}]=50 \mathrm{~ms} / 2500 \mathrm{~ms}$; flip angle $=90^{\circ} ; 80 \times 80$ matrix, in-plane voxel size $=3.124 \mathrm{~mm} \times 3.124 \mathrm{~mm}$; slice thickness $=6 \mathrm{~mm}$ [no gap]; 25 trans-axial slices per volume; interleaved slice acquisition). Four additional GE-EPI excitations were performed before the task began to allow transverse magnetization to approach its steady-state value; the data for these excitations were discarded. A T1-weighted spoiled gradient image was acquired from each participant for spatial normalization purposes $\left(\mathrm{TE} / \mathrm{TR}=3 \mathrm{~ms} / 25 \mathrm{~ms}\right.$; flip angle $=45^{\circ}, 256 \times 256$ matrix; in-plane voxel size $=0.781 \mathrm{~mm} \times 0.781 \mathrm{~mm}$; slice thickness $=1.5 \mathrm{~mm}$ [no gap]; 124 trans-axial slices per volume).

\subsection{Behavioral data analysis}

2.5.1 Tracking Error-Root-mean-square (RMS) of the tracking error was calculated using the formula sqrt [mean $\left.\left(\mathrm{x}^{2}+\mathrm{y}^{2}\right)\right]$, where $\mathrm{x}$ and $\mathrm{y}$ are the horizontal and vertical cursor distances at each time point, respectively, from the center of the screen in pixels.

2.5.2 Test for overall dual-tasking by SD interaction-The mean tracking error in each 2 min tracking trial was examined in a repeated-measures analysis of variance (ANOVA) with SD (pre/post), Condition (single-/dual-task), Block (1-3), and Trial (1/2) as the within-subject factors. The effect of interest was the interaction between SD and 
Condition. If dual-tasking lessens the SD effect, the mean tracking error should be lower in the post SD dual-task condition than in the single-task condition. P-values for factors that did not satisfy the sphericity assumption were corrected using the Huynh-Feldt correction.

2.5.3 Test for dual-response processing by SD interaction-To test the effects of sleep deprivation on the response component of dual-tasking, tracking error was binned time-locked to the detection stimulus onset for both targets and non-targets as separate timeseries data. For both data sets, tracking error was divided into $640 \mathrm{~ms}$ bins from $8000 \mathrm{~ms}$ before to $8000 \mathrm{~ms}$ after the event of interest (stimulus onset or response). This interval was chosen because $8000 \mathrm{~ms}$ was the duration of each target stimulus presentation during which only one target stimulus was on the screen. Bin width of $640 \mathrm{~ms}$ divided the $16000 \mathrm{~ms}$ window of interest into 25 bins. Binned time-series data were calculated separately for targets and non-targets within both the single- and the dual-task conditions. Even though detection was not performed in the single-task condition, presentation of detection stimuli served as control for visual excitation during the dual-task condition. Binned error for nontargets only included circle color changes that did not overlap with a target.

Stimulus-locked tracking error time-series data were analyzed with repeated-measures ANOVA consisting of SD (pre/post), Condition (single-/dual-task), detection stimulus types (target/non-target), Block (1-3), Trial (1-2), and Bin (1-25). If dual-response alleviates SD effect, the interaction among SD, Condition, and detection stimulus type would be significant. Specifically, since dual-response was only required for dual-task targets but not for non-targets, the tracking error was hypothesized to be lower for duals-task target than for dual-task non-target in post SD while the opposite pattern would be observed pre SD as was reported in Gazes et al. (2010). Binned tracking error analysis was also performed on the binned error time-locked to the detection response in a repeated-measures ANOVA with the factors SD, Block, Trial, and Bin. The factors Condition and detection stimulus types were not included because responses were only made to dual-task targets. Tracking error means were $\log$ transformed to satisfy the normality assumption of parametric tests. P-values for factors that did not satisfy the sphericity assumption were corrected using the Huynh-Feldt correction.

2.5.4 Visual detection variables-Two detection variables, RT and misses were recorded and analyzed in repeated-measures ANOVA models. Misses were the number of times, during a 2 min, tracking trial that the target disappeared before the participant responded. The factors in the ANOVA model included SD, Block, and Trial. P-values for factors that did not satisfy the sphericity assumption were corrected using the Huynh-Feldt correction.

\section{6 fMRI statistical analysis}

2.6.1 Preprocessing-All image preprocessing and analysis were implemented using the SPM5 software package (Wellcome Department of Cognitive Neurology) and other inhouse code written in MATLAB 7.1 (Mathworks, Natick, MA). The following procedures were used for each participant's GE-EPI data set. Data were temporally shifted to correct for the order of slice acquisition, using the first slice acquired in the TR as the reference. All GE-EPI images were realigned to the first volume of the first session and then coregistered to the T1-weighted (structural) image using mutual information. The structural image was then used to determine parameters for transformation into a standard space defined by the Montreal Neurologic Institute template brain supplied with Statistical Parametric Mapping (SPM5) (Friston, 2007). This transformation was then applied to the GE-EPI data, which were resliced using sine-interpolation to $2 \mathrm{~mm} \times 2 \mathrm{~mm} \times 2 \mathrm{~mm}$ and then spatially smoothed with an $8 \mathrm{~mm}$ full-width-at-half-maximum (FWHM) kernel. 
2.6.2 Time-series modeling-Voxel-wise general linear model (GLM) was performed on the fMRI data (Holmes \& Friston, 1998). In the first-level GLM, the GE-EPI time series were modeled with regressors representing the factors SD (pre/post), Condition (single-/ dual-task) and detection stimulus types (target/non-target) in each condition for each of the three blocks, plus a regressor representing the instruction screen for each block and additional regressors for false alarms and misses, which varied by block and subject. The regressors were constructed by convolutions of an indicator sequence (i.e. a train of discretetime delta functions) representing tracking and target/non-target event onsets, an assumed BOLD impulse response function (as represented by default in SPM5), and a rectangular function of duration dictated by the duration of the assumed neural response (Zarahn, 2000). In particular, correctly responded target stimulus regressors were modeled with event durations equaling to the respective RT (Grinband, Wager, Lindquist, Ferrera, \& Hirsch, 2008) to better capture the activation associated with the response.

In order to minimize the correlation between predictors in the time-series model, the nontarget onset predictors were orthogonalized with respect to the tracking predictor by regressing the non-target predictor with respect to the tracking predictor using the GramSchmidt orthogonalization technique. The tracking predictor contained the mean activation of the entire trial, which included not only activity related to tracking, but also any activity that persisted for most of the trial. Since non-targets occurred fairly frequently throughout the trial, it was unavoidable that the tracking block contained some variability associated with non-targets. For the non-target predictor, orthogonalization only removed the mean from the predictor, but the predictor still corresponded to the time-varying signal change associated with non-target event onsets, enabling valid interpretation of this predictor after, orthogonalization. The contrast estimate images were then used as the dependent variable in a second-level GLM.

2.6.3 Group analyses-Group GLM was performed on the two effects of interest based on the equivalent contrast to the corresponding interaction in the behavioral ANOVA analysis: (a) overall dual-task by SD interaction was tested with the contrast pre vs. post SD difference between dual- minus single-task tracking (algebraically represented as Pre[Dual Single] - Post[Dual - Single]); and (b) dual-response by SD interaction was tested with pre vs. post SD difference between dual-task target minus non-target (algebraically represented as Pre[Dual target - non-target] - Post[Dual target - non-target]). In addition, overall change in activation across SD was tested with pre vs. post SD sum of single- plus dual-task tracking (algebraically represented as Pre[Single + Dual] - Post[Single + Dual]). Except where mentioned, results were corrected for multiple comparisons across the whole brain using the Bonferroni correction for the number of resolution elements (Worsley et al., 1996), with an extent threshold of 20 contiguous voxels. Likely cytoarchitectonic labels for cluster maxima in these thresholded patterns were obtained using Talairach daemon software (Lancaster et al., 2000) and checked with visual inspection.

\section{Results}

\subsection{Behavioral analyses}

3.1.1 Effect of overall dual-tasking by SD interaction-Tracking error was tested in a repeated measures ANOVA containing the factors SD (pre/post), Condition (single-/dualtask), Block (1-3), and Trial (1-2). With SD, the mean tracking error increased from 20 pixels pre SD to 45 pixels post $\mathrm{SD}, F(1,17)=139.9, p<0.001$. All other main effects were not significant. Significant interaction was found between SD and Condition, $F(1,17)=$ $12.07, p=0.003$. The difference in tracking error between the single- and dual-task conditions followed opposite trends pre and post SD. As seen in Figure 2A, tracking error 
was higher in dual- than in single-task pre SD, whereas tracking error was slightly lower in the dual- than the single-task condition post SD. This observation suggests that dual-tasking enabled some resistance to the effects of SD. The significant interaction among SD, Block, and Trial $(F(2,34)=8.165, p=0.001)$, as well as the related interactions, SD and Block $(F(2,34)=10.71, p=0.002)$ and SD and Trial $(F(1,17)=11.26, p=0.004)$, were likely to reflect a learning effect pre SD and a time-on-task effect post SD in that overall tracking error decreased across the test sessions pre SD whereas the error increased across the test sessions post SD. All other interactions were not significant.

3.1.2 Dual-response by SD interaction-The interaction between dual-response processing and SD were tested by averaging tracking error in twenty-five $640 \mathrm{~ms}$ bins, timelocked to the onsets of target and non-targets in both single- and dual-task conditions. Binned tracking error was tested in a repeated-measures ANOVA with the factors SD, Condition, detection stimulus type (target/non-target), Block, Trial, and Bin (1-25). The SD main effect $(F(1,18)=106.4, p<0.001)$ and the interaction $\operatorname{SD} \times \operatorname{Block}(F(2,36)=8.916, p$ $=0.003$ ) were similar to those found in the overall dual-tasking by SD analysis. The Bin effect $(F(24,432)=2.112, p=0.035)$ was mainly driven by the increased error approximately $2 \mathrm{~s}$ after stimulus onset.

For post SD performance, lower error for dual-task targets than for dual-task non-targets would suggest an effect of dual-response on SD. The significant interactions among SD $\times$ Condition $\times$ detection stimulus type $\times \operatorname{Block}(F(2,36)=3.28, p=0.049), \mathrm{SD} \times$ Condition $\times$ detection stimulus type $\times \operatorname{Bin}(F(24,432)=4.66, p<0.001)$, and the corresponding lower order interactions, Condition $\times \operatorname{Bin}(F(24,432)=3.040, p=0.017)$, detection stimulus type $\times \operatorname{Bin}(F(24,432)=2.532, p=0.004), \operatorname{SD} \times$ detection stimulus type $(F(1,18)=16.24, p=$ $0.001), \mathrm{SD} \times$ detection stimulus type $\times \operatorname{Bin}(F(24,432)=3.069, \mathrm{p}=0.001)$, and Block $\times \operatorname{Bin}$ $(F(48,864)=1.851, p=0.024)$, were all significant. Figures $2 \mathrm{~B}, 2 \mathrm{C}$, and $2 \mathrm{D}$ show the two highest order interactions. Both figures displayed lower error for dual-task targets than for dual-task non-targets post SD, consistent with a dual-response effect. The interaction among $\mathrm{SD} \times$ Condition $\times$ detection stimulus type $\times$ Block showed that the dual-response effect was most pronounced in the third block of post SD while the interaction among SD $\times$ Condition $\times$ detection stimulus type $\times$ Bin revealed a temporal effect with respect to stimulus onset such that error was the lowest from 3 to $8 \mathrm{~s}$ after stimulus onset. Error for dual-task nontargets, however, was not lower than error for single-task non-targets, suggesting the lack of a dual-visual processing effect on SD.

Since dual-response processing was determined to be the factor associated with performance-improvement post SD, tracking error was binned, time-locked to response, to further examine the effect of dual-response. Figure $2 \mathrm{E}$ shows that after response to a target, improvement in the post SD error almost reached the pre SD error rate for about $6 \mathrm{~s}$. Thus, response complexity counteracted SD effects to the extent that performance almost returned to pre SD level. To tease out the time-on-task effect of dual-response on performance post SD, post SD data in Figure E was separated into 3 blocks in Figure F. The dual-response effect was the minimal in block 1 but as time-on-task increased, performance improvement associated with dual-response effect magnified in blocks 2 and 3 to the extent that time-ontask effect was eliminated in the short time period after dual-response as shown by the dip in blocks 2 and 3 intersecting with the curve for block 1 at a tracking error of about 25 pixels.

3.1.3 Visual detection variables-RT and the number of misses to targets in the dualtask condition were analyzed with repeated-measures ANOVA containing the factors SD, Block, and Trial. For RT, only the main effects, $\operatorname{SD}(F(1,18)=23.67, p<0.001)$ and Block $(F(2,36)=3.449, p=.044)$, were significant. RT increased from a mean of $1061 \mathrm{~ms}$ to 1699 $\mathrm{ms}$ post $\mathrm{SD}$, and increased from $1247 \mathrm{~ms}$ in Block 1 to $1557 \mathrm{~ms}$ in Block 2, then decreased 
to $1335 \mathrm{~ms}$ in Block 3. For misses, only the main effect $\mathrm{SD}$ reached significance, $F(1,18)=$ $8.683, p=0.009$. Significant interactions were between $\mathrm{SD} \times \mathrm{Block}, F(2,36)=4.848, p=$ 0.014 , and among $\mathrm{SD} \times$ Block $\times$ Trial, $F(2,36)=4.330, p=0.021$. The number of misses increased from $4.7 \%$ pre SD to $13.3 \%$ post $\mathrm{SD}$, but the interactions did not show any clear pattern across the factors.

\section{2 fMRI analyses}

3.2.1 Changes in activation post SD-Changes in activation associated with SD were evaluated by contrasting the mean activation during tracking (i.e. combining single- plus dual-task conditions) from pre to post SD. Table 1 lists the brain regions with altered taskrelated activation levels post vs. pre SD. The major areas of reduced activation included bilateral inferior parietal lobules, bilateral postcentral gyri, bilateral middle temporal gyri, bilateral supplementary motor area (SMA), and bilateral medial frontal gyri. Increased activation post SD was found in bilateral primary visual cortices.

3.2.2 Tests of dual-tasking and dual-response effects-Tests for overall dual-task effects on brain activation associated with SD did not reveal any significant regions. As shown in Figure 3 and Table 2, test of dual-response effect on brain activation changes associated with SD showed significantly greater activation differences post SD in the left cerebellum, left somatosensory cortex, left extrastriate cortex, bilateral precuneus, left middle frontal gyrus, and left motor cortex, where the middle frontal gyrus is the lateral area of BA 6, which is the premotor cortex.

\section{Discussion}

Using dual-task tracking, the interaction between dual-tasking and SD effects on tracking error was examined behaviorally and with functional neuroimaging. We predicted that if dual-tasking provides resistance to the effects of SD on performance, this would be evident as reduced tracking error post SD during dual-task targets relative to dual-task non-targets. Despite a mean increase in tracking error after SD, error time-locked to dual-task target was than error for dual-task non-target, and the overall dual-task error was slightly lower than single-task error as well. Thus, the behavioral result is consistent with the hypothesis that dual-response helps to reduce the impairment exerted by SD. fMRI analyses showed a greater activation difference between dual-task targets and non-targets post SD even though reduced overall activation was found after SD. The increased post SD difference was found in task-related regions.

\subsection{Interaction between dual-tasking and SD effects}

Our study results offer a resolution to the discrepancy among the previous multitask SD studies. The overall post SD error mean suggested performance impairment as presented in Caldwell's MATB result. However, a more interesting mechanism was revealed by the pattern of the stimulus-locked binned error means. Even though the overall performance was negatively affected by SD, the moments when dual-responding occurred, tracking performance actually improved dramatically, from a mean of 45 pixels to 25 pixels. The pre SD error mean was 20 pixels, only 5 pixels less than the error during dual-response.

Therefore, it is possible that if MATB scores had been recalculated time-locked to stimulus onset of the secondary tasks, the same performance improvement would have been shown at times of dual-responses. Our behavioral result suggests that having to perform two simultaneous motor responses helps to temporarily improve performance even under the debilitating effect of SD. This is consistent with Strangman et al.'s (2005) results that SD performance on a dual-joystick-controlled navigation task was comparable to pre SD performance. The task required moving two joysticks simultaneously, one in each hand, to 
control the flight an aircraft. Thus the demand on dual-motor response was high throughout the task, which protected performance from SD effects.

Consistent with the behavioral result, a similar pattern of change was found in brain activation. Overall, brain activation decreased post SD as was found by most SD studies (Chuah, et al., 2006; Thomas, et al., 2000) and followed the overall decrease in performance post SD. In contrast, activation changes associated with dual-response showed that the activation difference between dual-task targets and non-targets was greater post than pre SD. In other words, using the non-target activation as baseline for the respective day, the increase in activation for targets post SD was greater than the increase pre SD.

Most of the regions showing greater activation difference between targets and non-targets post than pre SD were associated with motor processes for the tracking task. The left precentral and postcentral gyri are part of the primary motor and somatosensory cortices respectively, which control motor execution of and haptic feedback from the right hand - the tracking hand (Flanders, 2005). The left middle frontal gyrus, Brodmann Area 6, is part of the premotor cortex, associated with motor planning (Halsband \& Lange, 2006). The middle occipital gyrus is associated with visual processing (Bullier \& Nowak, 1995). The increased activation of these tracking-related regions post SD replicates result reported by Strangman et al. (2005) in their dual-joystick navigation task and extends their finding by pinpointing dual-response to be a factor that alleviates SD effects.

\subsection{Time-on-task effect during SD}

The overall mean of the tracking error shows a time-on-task effect post consistent with previous tracking tasks (Bohnen \& Gaillard, 1994; Caldwell \& Ramspott, 1998). However, the ameliorating effect of dual-responding increased as the time-on-task effect worsened. Figure $2 \mathrm{~F}$ shows that while the overall tracking error for blocks 2 and 3 were greater, the two curves returned to the error level in block 1 after dual-response, suggesting that the arousing nature of dual-response fully counteracted time-on-task effect during SD.

However, under the current task design, the dual-response effect is only intermittent, as seen in the overall increase in the mean error across the blocks and the minimal improvement in the dual-task error mean in Figure 2A. This is consistent with the intermittent appearance of targets in each trial (only 8 per trial). Increasing the number of dual-response should result in greater overall performance improvement.

\subsection{Mechanism of dual-response for alleviating SD effects on performance}

Under well-rested conditions, dual-tasking has been found to recruit prefrontal activation, presumably for resolving bottlenecks. Bottlenecks cause processing conflicts due to competition for limited resources (Schubert \& Szameitat, 2003). According to t Schubert and Szameitat (2003), in the occurrence of a bottleneck (e.g. response selection processing), prefrontal activation enforces task order control which proportions attention to the more immediate task, thus providing the task with processing priority. Indeed, in the pre SD analyses of our dual-task tracking study, we reported evidence for the bottleneck stage to reside in dual-responding (Gazes et al., 2010). Interestingly, post SD error during dualresponse was the lowest relative to other conditions. In other words, whereas error was the highest during dual-response pre error was the lowest during dual-response post SD when compared to the baseline conditions in each respective day.

The contradictory results between pre and post SD may not be merely coincidental. Dualtasks may help to resist the effects of SD better than single-tasks because the task order control needed in dual-tasking engages the prefrontal cortex (PFC) more than single-tasks. Pilcher et al. (2007) used the Controlled Attention model to argue that engaging tasks that 
activate working memory and attention processes contribute to greater wakefulness (Kane \& Engle, 2002; Pilcher, et al., 2007). A possible mechanism by which engaging the prefrontal cortex may increase wakefulness is by the inhibition of sleep initiation (Datta \& Maclean, 2007). Additional evidence of the association between PFC activity and wakefulness was found in the middle frontal gyrus in which its activation level correlated negatively with sleepiness in sleep restricted participants while performing the n-back task (Honma, Soshi, Kim, \& Kuriyama, 2010). Our imaging result shows consistent effect in the dual-response comparison such that the middle frontal gyrus was among the regions with greater activation to dual-response post SD. These results suggest that the mechanism through which dualresponse resists SD effects involves activation of the PFC, which is likely to actively inhibit sleep initiation.

\subsection{Study limitations and future directions}

A major limitation to the current study was the lack of a counterbalanced design for the order of pre and post SD testing. Thus, it is possible that the effects are confounded with learning and other order effects, but it is unlikely that these potential confounds changed the effects of interest reported in this paper since the comparisons were made with performance on the same day. The second limitation was the possible long term effect exerted by rTMS treatment on fMRI activation. Even though the TMS targets were selected to affect an unrelated task, it was possible for the effect to extend to the current task. However, analysis not reported here showed that rTMS did not show any significant effect on the task manipulations reported in the current study. A third limitation was the mixture of impulse and RT modeled regressors in the GLM analysis on the fMRI data. Ideally, events are modeled with the exact duration in the GLM, but since it is not possible to know the exact neural duration of each event, the best possible approximations were used. For targets, reaction times were used under the assumption that neural activity persisted at least until a response was made. For non-targets, ideally we have some signal that could inform us when the processing of the non-target is complete, but in the real world, we could only use an impulse to approximate the duration. The last limitation was mentioned in the Methods section 2.6.2. In order to minimize the correlation between the tracking and the non-target regressors in the GLM, the non-target regressors were orthogonalized with respect to the tracking regressor. However, the orthogonalization only had the effect of removing the mean from the regressor while retaining the variability around the mean. Thus, the orthogonalization most likely did not change the interpretability of the regressor.

Our study showed that intermittent dual-response may alleviate performance impairment under SD, but the beneficial effect needs further investigation to better characterize the nature of the improvement. Future studies should explore the duration of the benefit of inserting a demanding motor task to increase alertness and how well the beneficial effect extends to real world tasks such as driving.

- Highlights

- Dual-response alleviated performance degradation post sleep deprivation.

- Dual-response was associated with greater brain activation difference post SD.

- Greater brain activation difference found in task-related regions post SD.

\section{Acknowledgments}

This work was supported by Army Research Office and the Defense Advanced Research Projects Agency (ARO and DARPA Grant DAAD 19-02-1-0047). The views, opinions, and/or findings contained in this article/ 
presentation are those of the author/presenter and should not be interpreted as representing the official views or policies, either expressed or implied, of the Defense Advanced Research Projects Agency or the Department of Defense. We thank Oksana Tatarina-Nulman, Linda Sanders, and Gray Herzberg for their help in data collection.

\section{References}

Bell-McGinty S, Habeck C, Hilton HJ, Rakitin B, Scarmeas N, Zarahn E, et al. Identification and differential vulnerability of a neural network in sleep deprivation. Cereb Cortex. 2004; 14(5):496502. [PubMed: 15054065]

Bohnen HG, Gaillard AW. The effects of sleep loss in a combined tracking and time estimation task. Ergonomics. 1994; 37(6):1021-1030. [PubMed: 8026449]

Brown SW. Attentional resources in timing: interference effects in concurrent temporal and nontemporal working memory tasks. Perception \& Psychophysics. 1997; 59(7):1118-1140. [PubMed: 9360484]

Bullier J, Nowak LG. Parallel versus serial processing: new vistas on the distributed organization of the visual system. Current Opinion in Neurobiology. 1995; 5(4):497-503. [PubMed: 7488852]

Caldwell JA, Ramspott S. Effects of task duration on sensitivity to sleep deprivation using multiattribute task battery. Behavioral Research Methods, Instruments, \& Computers. 1998; 30(4):651660.

Chuah YM, Venkatraman V, Dinges DF, Chee MW. The neural basis of interindividual variability in inhibitory efficiency after sleep deprivation. J Neurosci. 2006; 26(27):7156-7162. [PubMed: 16822972]

Datta S, Maclean RR. Neurobiological mechanisms for the regulation of mammalian sleep-wake behavior: reinterpretation of historical evidence and inclusion of contemporary cellular and molecular evidence. Neurosci Biobehav Rev. 2007; 31(5):775-824. [PubMed: 17445891]

Donnell JM. Performance decrement as a function of total sleep loss and task duration. Perceptual \& Motor Skills. 1969; 29(3):711-714. [PubMed: 5365071]

Drummond SP, Brown GG, Gillin JC, Strieker JL, Wong EC, Buxton RB. Altered brain response to verbal learning following sleep deprivation. Nature. 2000; 403(6770):655-657. [PubMed: 10688201]

Drummond SP, Brown GG, Strieker JL, Buxton RB, Wong EC, Gillin JC. Sleep deprivation-induced reduction in cortical functional response to serial subtraction. Neuroreport. 1999; 10(18):37453748. [PubMed: 10716202]

Durmer JS, Dinges DF. Neurocognitive consequences of sleep deprivation. Semin Neurol. 2005; 25(1):117-129. [PubMed: 15798944]

Flanders M. Functional somatotopy in sensorimotor cortex. Neuroreport. 2005; 16(4):313-316. [PubMed: 15729128]

Friston, KJ. Statistical parametric mapping : the analysis of functional brain images. London: Academic; 2007.

Gazes Y, Rakitin BC, Steffener J, Habeck C, Butterfield B, Ghez C, et al. Performance degradation and altered cerebral activation during dual performance: evidence for a bottom-up attentional system. Behavioural Brain Research. 2010; 210(2):229-239. [PubMed: 20188768]

Grinband J, Wager TD, Lindquist M, Ferrera VP, Hirsch J. Detection of time-varying signals in eventrelated fMRI designs. Neuroimage. 2008; 43(3):509-520. [PubMed: 18775784]

Habeck C, Rakitin BC, Moeller J, Scarmeas N, Zarahn E, Brown L, et al. An event-related fMRI study of the neurobehavioral impact of sleep deprivation on performance of a delayed-match-to-sample task. Brain Research Cognitive Brain Research. 2004; 18(3):306-321. [PubMed: 14741317]

Halsband U, Lange RK. Motor learning in man: a review of functional and clinical studies. Journal of Physiology Paris. 2006; 99(4-6):414-424.

Holmes A, Friston K. Generalisability, random effects and population inference. Neuroimage. 1998; 7:S754.

Honma M, Soshi T, Kim Y, Kuriyama K. Right prefrontal activity reflects the ability to overcome sleepiness during working memory tasks: a functional near-infrared spectroscopy study. PLoS One. 2010; 5(9):el2923. 
Horne JA, Anderson NR, Wilkinson RT. Effects of sleep deprivation on signal detection measures of vigilance: implications for sleep function. Sleep. 1983; 6(4):347-358. [PubMed: 6665397]

Jolicoeur P. Restricted attentional capacity between sensory modalities. Psychonomics Bulletin and Review. 1999; 6(1):87-92.

Kane MJ, Engle RW. The role of prefrontal cortex in working-memory capacity, executive attention, and general fluid intelligence: an individual-differences perspective. Psychon Bull Rev. 2002; 9(4):637-671. [PubMed: 12613671]

Lancaster JL, Woldorff MG, Parsons LM, Liotti M, Freitas CS, Rainey L, et al. Automated Talairach atlas labels for functional brain mapping. Human Brain Mapping. 2000; 10(3):120-131. [PubMed: 10912591]

National Sleep Foundation. Sleep in America Poll. Arlington, VA: National Sleep foundation; 2011.

Ogawa S, Menon RS, Tank DW, Kim SG, Merkle H, Ellermann JM, et al. Functional brain mapping by blood oxygenation level-dependent contrast magnetic resonance imaging. A comparison of signal characteristics with a biophysical model. Biophysical Journal. 1993; 64(3):803-812. [PubMed: 8386018]

Pace-Schott EF, Hutcherson CA, Bemporad B, Morgan A, Kumar A, Hobson JA, et al. Failure to find executive function deficits following one night's total sleep deprivation in university students under naturalistic conditions. Behavioral Sleep Medicine. 2009; 7(3):136-163. [PubMed: 19568965]

Pashler H. Dual-task interference in simple tasks: data and theory. Psychological Bulletin. 1994; 116(2):220-244. [PubMed: 7972591]

Pilcher JJ, Band D, Odle-Dusseau HN, Muth ER. Human performance under sustained operations and acute sleep deprivation conditions: toward a model of controlled attention. Aviation, Space, and Environmental Medicine. 2007; 78(5 Suppl):B15-B24.

Pilcher JJ, Huffcutt AI. Effects of sleep deprivation on performance: a meta-analysis. Sleep. 1996; 19(4):318-326. [PubMed: 8776790]

Pruessmann KP, Weiger M, Scheidegger MB, Boesiger P. SENSE: sensitivity encoding for fast MRI. Magnetic Resonance in Medicine. 1999; 42(5):952-962. [PubMed: 10542355]

Schubert T, Szameitat AJ. Functional neuroanatomy of interference in overlapping dual tasks: an fMRI study. Cognitive Brain Research. 2003; 17(3):733-746. [PubMed: 14561459]

Strangman G, Thompson JH, Strauss MM, Marshburn TH, Sutton JP. Functional brain imaging of a complex navigation task following one night of total sleep deprivation: a preliminary study. Journal of Sleep Research. 2005; 14(4):369-375. [PubMed: 16364137]

Thomas M, Sing H, Belenky G, Holcomb H, Mayberg H, Dannals R, et al. Neural basis of alertness and cognitive performance impairments during sleepiness. I. Effects of $24 \mathrm{~h}$ of sleep deprivation on waking human regional brain activity. J Sleep Res. 2000; 9(4):335-352. [PubMed: 11123521]

Worsley KJ, Marrett S, Neelin P, Vandal AC, Friston KJ, Evans AC. A unified statistical approach for determining significant signals in images of cerebral activation. Human Brain Mapping. 1996; 4(1):58-73. [PubMed: 20408186]

Zarahn E. Testing for neural responses during temporal components of trials with BOLD fMRI. Neuroimage. 2000; 11(6 Pt 1):783-796. [PubMed: 10860802] 
A

Track

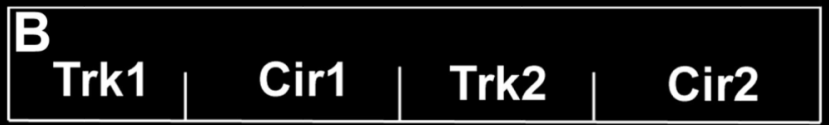

Track

O

Figure 1.

(A) Screenshot of the task showing tracking stimulus at the center and visual detection stimulus at the four corners. The small circle to the lower right of the plus sign is the tracking cursor controlled by participants. Colored circles for the visual detection task can be any of four colors: red, green, blue, and yellow. The two matching blue circles constitute a target and the non-matching red and yellow circles are non-targets. The word "Track" on either side of screen signals a single-task condition while "Circles" (not shown) signals a dual-task condition. (B) The structure of a block, consisting of 2 trials of each condition. Single-task is shown as Trkl and Trk2, and dual-task as Cirl and Cir2. The order of trials shown is an example. The trial order is randomized for each block. 
A

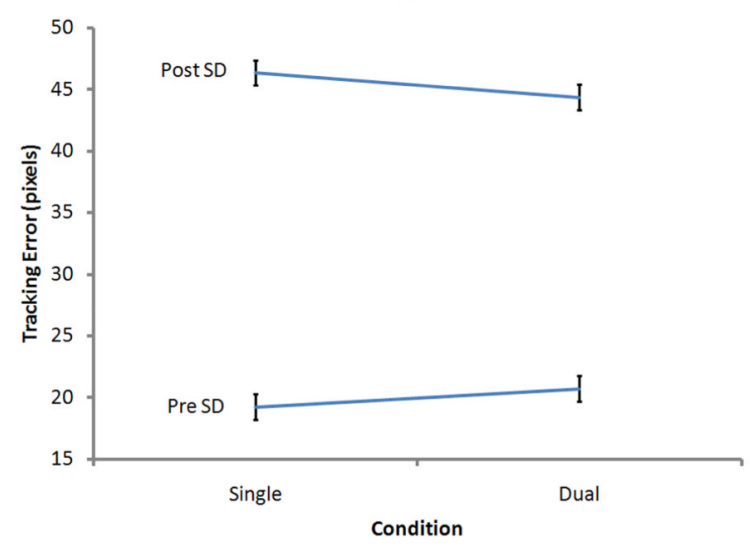

$\mathrm{C}$

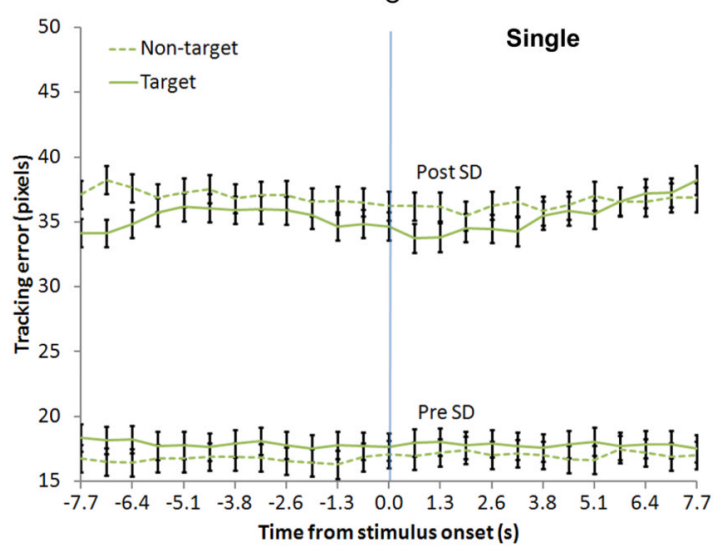

$\mathrm{E}$

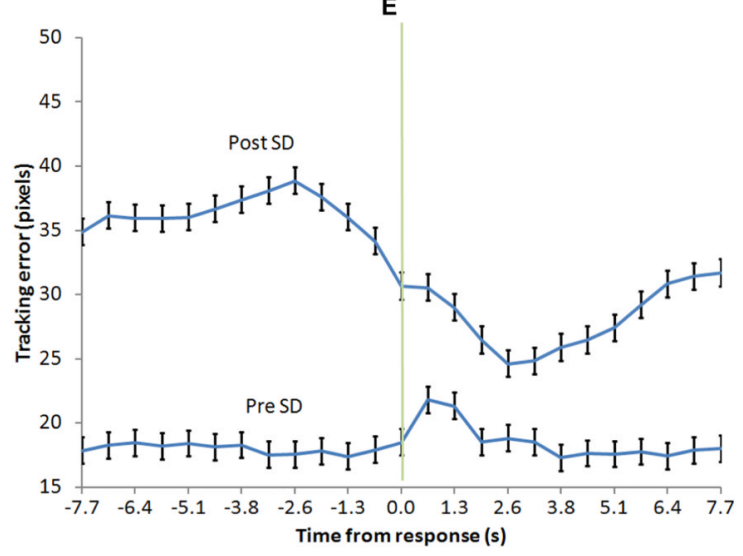

B
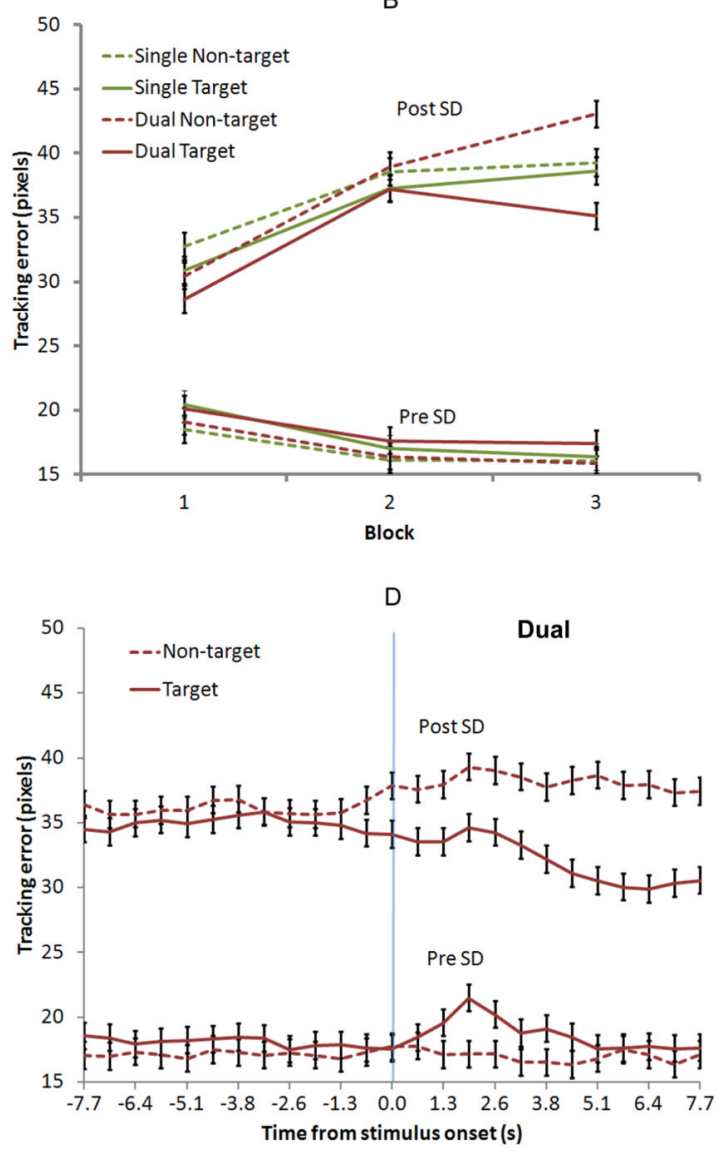

$\mathbf{F}$

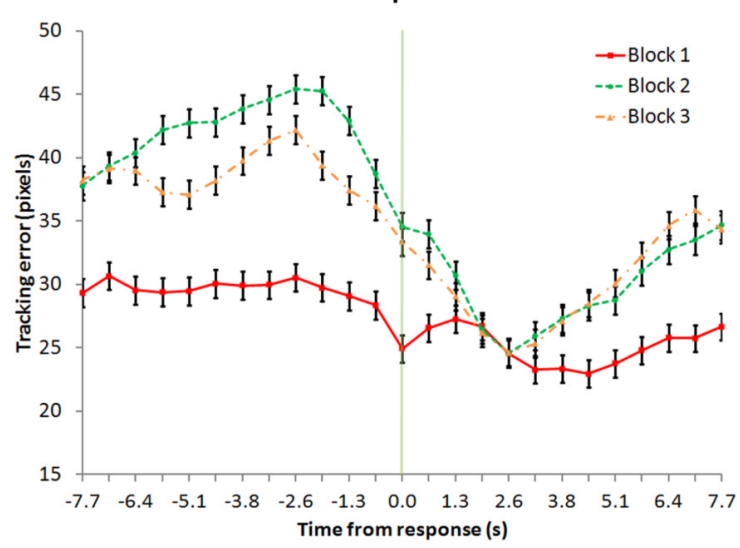

Figure 2.

Plots of tracking error pre and post SD: (A) in the single- and the dual-task conditions, (B) across three blocks for targets and non-targets in the single- and the dual-task conditions, (C) and (D) across 25 time bins time-locked to stimulus-onset for targets and non-targets in the single and the dual-task conditions, (E) across 25 time bins time-locked to response-onset for dual-task targets, and (F) across 25 time bins time-locked to response-onset for dual-task targets for each of the three blocks. Vertical lines in C, D, E, and F mark the event-onset times. Error bars represent standard errors. 


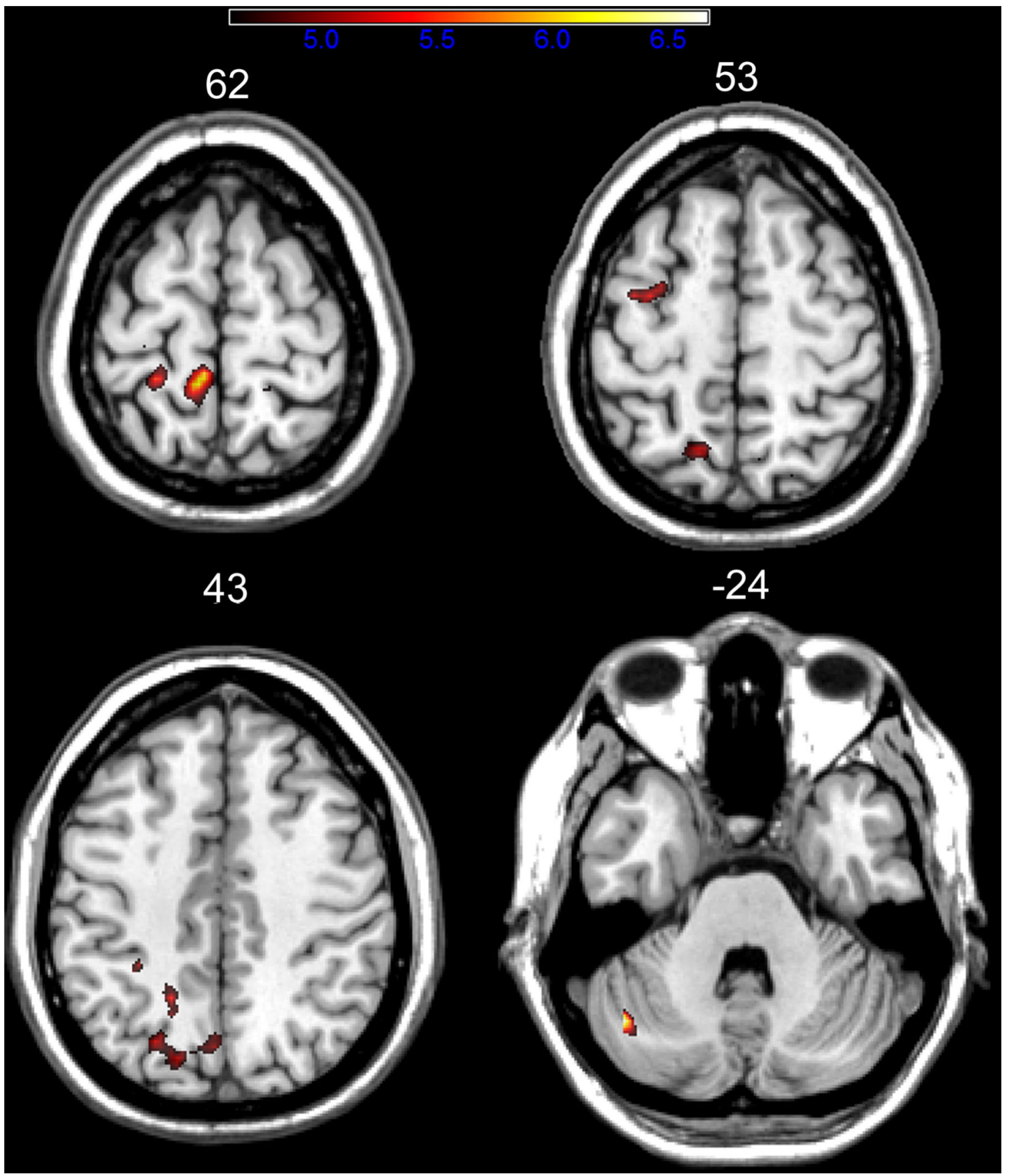

Figure 3.

Axial slices of regions with significantly greater post SD activation difference between dualtask targets and non-targets than the pre SD difference. Image was thresholded at $p<0.05$, Bonferroni corrected for the number of resels. White numbers indicate Talairach $\mathrm{z}$ coordinate. 


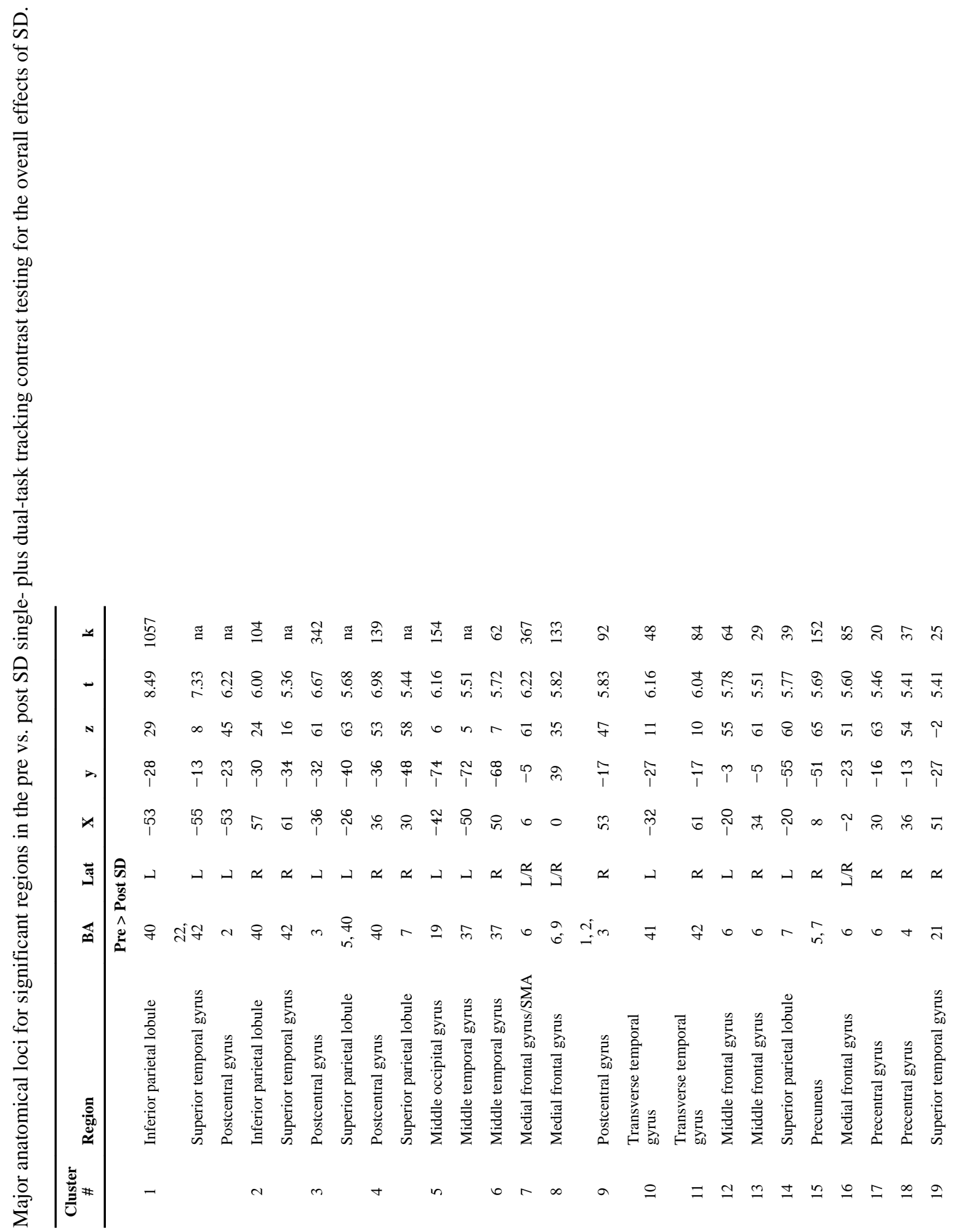




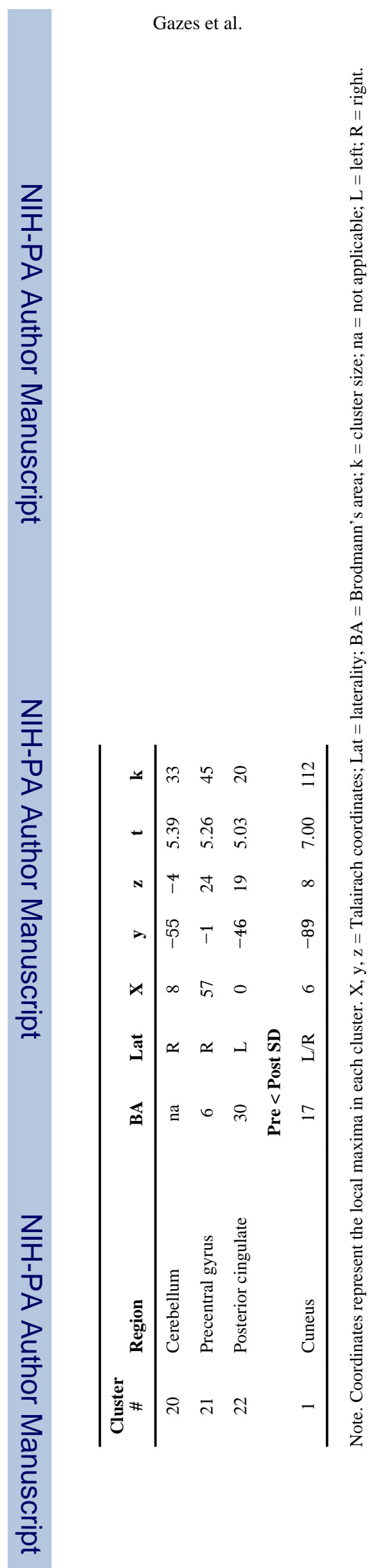

Brain Cogn. Author manuscript; available in PMC 2013 April 1. 


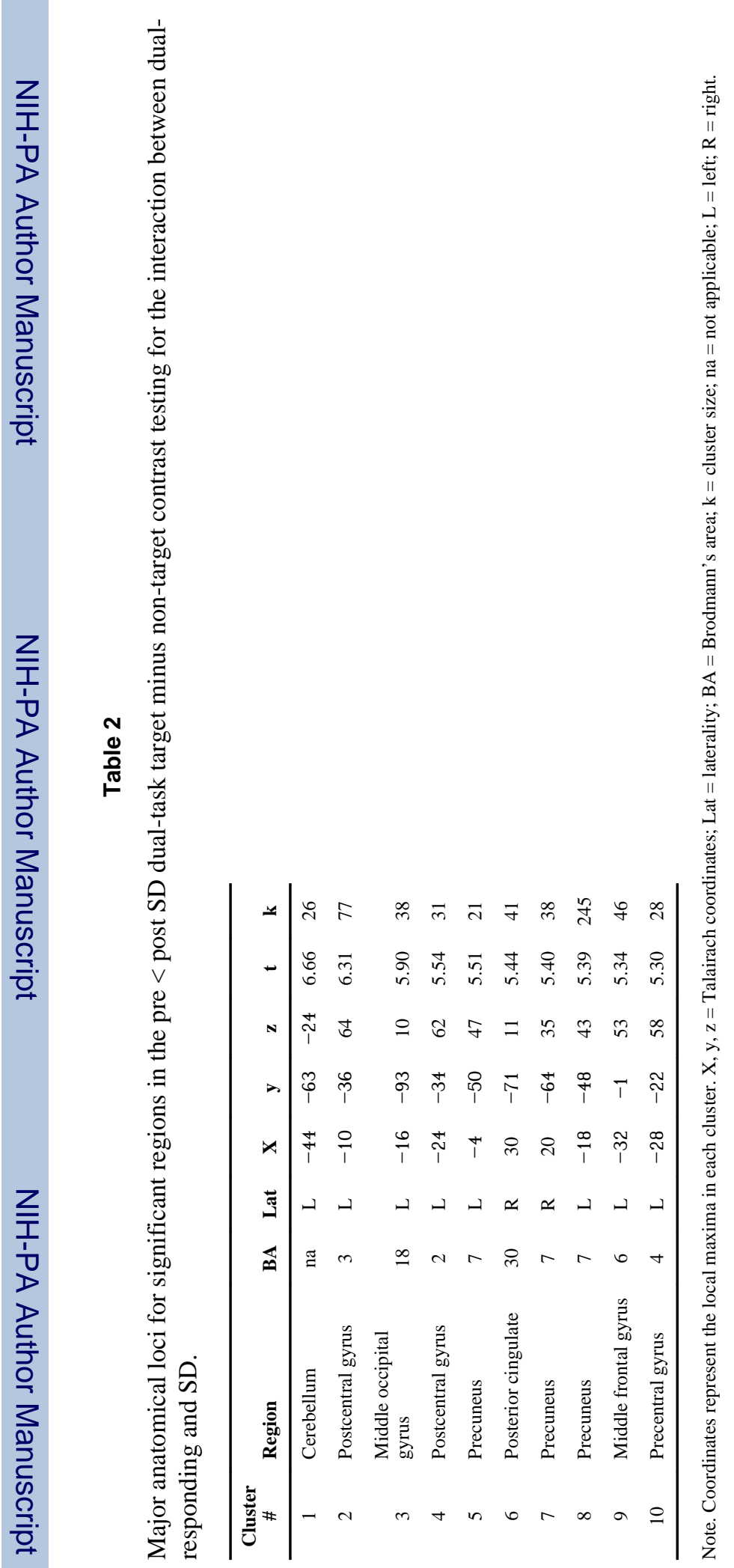

Brain Cogn. Author manuscript; available in PMC 2013 April 1. 